\title{
BMJ Open Use of healthcare services and assistive devices among centenarians: results of the cross-sectional, international 5-COOP study
}

\author{
Julien Dupraz (10 , ${ }^{1}$ Karen Andersen-Ranberg, ${ }^{2,3}$ Stefan Fors, ${ }^{4}$ Marie Herr, ${ }^{5,6,7}$ \\ Francois R Herrmann, ${ }^{8}$ Tomoko Wakui, ${ }^{9}$ Bernard Jeune, ${ }^{2}$ Jean-Marie Robine, ${ }^{10}$ \\ Yasuhiko Saito, ${ }^{11}$ Brigitte Santos-Eggimann, ${ }^{1}$ On behalf of the 5-COOP group
}

To cite: Dupraz J, AndersenRanberg K, Fors S, et al. Use of healthcare services and assistive devices among centenarians: results of the cross-sectional, international 5-COOP study. BMJ Open 2020;10:e034296. doi:10.1136/ bmjopen-2019-034296

- Prepublication history and additional material for this paper are available online. To view these files, please visit the journal online (http://dx.doi. org/10.1136/bmjopen-2019034296).

Received 13 September 2019 Revised 10 February 2020 Accepted 19 February 2020

Check for updates

(C) Author(s) (or their employer(s)) 2020. Re-use permitted under CC BY-NC. No commercial re-use. See rights and permissions. Published by BMJ.

For numbered affiliations see end of article.

Correspondence to

Dr Julien Dupraz;

julien.dupraz@unisante.ch

\section{ABSTRACT}

Objectives To measure the use of healthcare services and assistive devices by centenarians in five countries.

Design Cross-sectional study using a survey questionnaire.

Setting Community-dwelling and institutionalised centenarians living in Japan, France, Switzerland, Sweden and Denmark.

Participants 1253 participants aged 100 or in their 100th year of life, of whom 1004 (80.1\%) were female and 596 $(47.6 \%)$ lived in institutions.

Main outcome measures Recent use of medical visits, nursing care at home, home-delivered meals, acute care hospital stays overnight, professional assessments such as sight tests, mobility aids and other assistive devices. A set of national healthcare system indicators was collected to help interpret differences between countries.

Results There was considerable variability in the healthcare services and assistive devices used by centenarians depending on their country and whether they were community-dwelling or institutionalised. In contrast to the relatively homogeneous rates of hospitalisation in the past year (around 20\%), community-dwelling centenarians reported widely ranging rates of medical visits in the past 3 months (at least one visit, from $32.2 \%$ in Japan to $86.6 \%$ in France). The proportion of community-dwellers using a mobility device to get around indoors (either a walking aid or a wheelchair) ranged from $48.3 \%$ in Japan to $79.2 \%$ in Sweden. Participants living in institutions and reporting the use of a mobility device ranged from $78.6 \%$ in Japan to $98.2 \%$ in Denmark. Conclusions Our findings suggest major differences in care received by centenarians across countries. Some may result from the characteristics of national healthcare systems, especially types of healthcare insurance coverage and the amounts of specific resources available. However, unexplored factors also seem to be at stake and may be partly related to personal health and cultural differences.

\section{INTRODUCTION}

Due to declining birth rates and rising life expectancy, the segment of the world's population aged 60 years or more is the fastest
Strengths and limitations of this study

- This is the first international investigation of healthcare services use by centenarians using a common survey questionnaire, thus enabling direct comparisons between countries with similar levels of economic development.

- We included previously unexplored fields such as the delivery of meals at home, the frequency of dental and sensory assessments and the use of assistive devices.

- Participation rates, sampling methods and interview modes varied according to settings, which may have differentially affected the representativeness of the participants in each country.

- Differences in healthcare services use by centenarians in these countries may have been due partly to variations in the involvement of informal carers-a dimension we were unable to capture.

growing. ${ }^{1}$ In high-income countries, the oldest-old group (aged $\geq 85$ years) is the most rapidly growing. ${ }^{2}$ Centenarians offer a spectacular demonstration of this phenomenon: estimated to be less than half a million worldwide in 2015, they are expected to exceed 21 million in $2100 .{ }^{13}$ Consequently, the last few decades have seen a growing number of centenarian studies, with diverse purposes and methods. ${ }^{4-6}$

Despite common low mortality rates and comparable economic conditions, Japan, France, Switzerland, Sweden and Denmark show varying degrees of mortality selection among their oldest-old. ${ }^{7}$ It is milder in Japan, which shows the fastest increase in the number of centenarians, intermediate in France and Switzerland, and stronger in Sweden and Denmark where the rate of increase is the lowest. The 5-Country Oldest Old Project (5-COOP) was set up to assess and 
compare the sociodemographic characteristics, health status, living conditions and healthcare services use of people living in these countries who were exactly 100 years of age. ${ }^{78}$

The resource needs induced by population ageing challenge today's social and healthcare systems. ${ }^{2}$ However, research dealing specifically with healthcare services used by centenarians remains rare and has shown heterogeneous results. ${ }^{9-11}$ Moreover, international comparisons using common methods are inexistent. Lack of information prevents any anticipation of healthcare needs or the design of policies dedicated to this vulnerable population. Taking advantage of data collected by 5 -COOP, the present paper aims to quantify and compare the use of healthcare services and assistive devices at the age of 100 in the five countries involved. We do not discuss potential explanatory factors such as morbidity, disability and cultural habits, nor do we consider the appropriateness of use. However, we do present comparative information on national healthcare systems, extracted from published international statistics, in order to give our results the perspective provided by shared indicators.

\section{METHODS}

\section{Study design and population}

5-COOP was a cross-sectional international study conducted in Japan, France, Switzerland, Sweden and Denmark. Inclusion criteria were residency (communitydwelling or institutionalised) of one of the five countries and being 100 years of age or in one's 100th year of life at enrolment. Individuals who were hospitalised when first contacted or did not speak the language of their country of residence were excluded. The intended sample size was 250 participants in each country. Between 2011 and 2014, participants were recruited via municipal lists in Japan (selected municipalities in five prefectures), regional lists in France (Languedoc-Roussillon region) and Switzerland (French-speaking region), and national lists in Sweden and Denmark. Overall, $54.5 \%$ of centenarians contacted agreed to participate, ranging from $30.6 \%$ in France to $85.6 \%$ in Sweden. The Swedish team oversampled male participants to compensate partially for the imbalanced sex ratio. One thousand two hundred fiftythree centenarians were finally included (346 in Japan, 212 in France, 170 in Switzerland, 274 in Sweden and 251 in Denmark).

\section{Data collection}

The 5-COOP data collection protocol involved the administration of a questionnaire and, when possible, a physical examination. A common generic questionnaire containing standardised instruments was first designed in English and then translated into local languages by national investigators. Back-translation was used to ensure the quality of the process, and translated questionnaires were pretested before their use with participants. The questionnaire contained a core of compulsory questions, supplemented by optional ones which country teams were free to include or not. Overall, investigators were able to carry out face-to-face visits in $73.3 \%$ of cases $(42.9 \%$ in France, $58.8 \%$ in Sweden, $70.8 \%$ in Japan and $100 \%$ in Switzerland and Denmark). The remaining participants were contacted by telephone $(14.5 \%$ of total, mostly in Japan and Sweden) or were sent a postal questionnaire (12.2\% of total, mostly in France). Centenarians (either alone or assisted by a proxy) participated actively in two-thirds of all the interviews $(42.5 \%$ in Japan, $59.4 \%$ in France, $62.8 \%$ in Sweden, $85.9 \%$ in Switzerland and $96.8 \%$ in Denmark), with information being provided by proxies in the remaining cases. The most frequent reason for a proxy interview was cognitive decline (36.0\%), followed by hearing impairment, general weakness, altered consciousness or speech $(10.5 \%$ each) and anxiety $(0.8 \%)$. In $31.8 \%$ of cases, there was another reason for a proxy interview or the reason was unknown.

\section{Use of healthcare services and assistive devices}

The present study was based on healthcare information reported in the questionnaire (corresponding sections are provided in online supplementary file 1), including the use of the following services:

- Medical visits (no specialty specified) during the previous 3 months (community-dwelling participants only);

- Nursing care at home during the previous 4 weeks (community-dwelling participants only);

- Delivery of meals at home during the previous 4 weeks (community-dwelling participants only);

- Acute care hospital stays overnight (including in an emergency room or psychiatric/psychogeriatric care) during the previous 12 months (all participants).

Use of each service was dichotomised as present or absent.

The last times which centenarians underwent a professional assessment of oral health, their eyes and vision and their hearing were dichotomised as within the previous 2 years or not (ie, longer ago or never). The last times centenarians had their blood pressure measured, their weight taken and their needs for nursing care, home help and social care evaluated was dichotomised as within the previous year or not.

Lastly, the use of a mobility device for moving around indoors and outdoors was recorded, as were the possession of glasses or contact lenses, hearing aids, dentures, bath chairs, elevated toilet seats, handles, banisters, lifting poles, medical beds and alarms.

\section{Statistics}

This work was based on descriptive statistics. Overall and country-specific proportions are reported with their respective $95 \%$ CIs and, inspired by a recent publication by Papanicolas et al, ranked in decreasing order using a colour code for each country. ${ }^{12}$ Stratification was applied according to place of living (community vs institution). Results from Sweden were weighted to account for the 
oversampling of male participants. As some questions suffered from a relatively high occurrence of missing data, we indicate when the results were based on data from fewer than 30 respondents. A detailed table with the number of respondents for each item is provided in online supplementary file 2 . The use of statistical tests was not considered relevant in this setting. Statistical analysis was performed using Stata/IC V.15.1.

\section{Specific characteristics of national healthcare systems}

To help interpret differences between countries, we developed a list of healthcare system indicators relevant to the issues being studied and documented in most countries, including older-population demography and healthcare expenditure, financing, resources and use. The OECD Health Statistics and Health System Characteristics Survey and the UN World Population Prospects were used to ensure that national measurements were as comparable as possible. The year 2015 was chosen because it provided the most recent validated data across all countries and it was immediately after the 5-COOP data collection process. Information unavailable from these sources was obtained from national data sources when possible and relevant.

\section{Patient and public involvement}

There was no direct participation of patients or the public in the design or conduct of this study.

\section{RESULTS}

\section{Study sample}

Of the 1253 participants, $1004(80.1 \%)$ were female, ranging between $77.7 \%$ in Denmark, $82.7 \%$ in Japan, $83.5 \%$ in France and $86.5 \%$ in Switzerland. In Sweden, the crude proportion of female participants was $72.6 \%$, corresponding to $81.9 \%$ after weighting to account for the oversampling of male participants. The proportions of participants living in institutions were comparable across countries $(41.6 \%$ in Japan, $42.3 \%$ in Sweden, $44.3 \%$ in France and $45.8 \%$ in Denmark), with the notable exception of Switzerland, where this share was substantially higher $(74.7 \%)$.

\section{Use of healthcare services}

The proportion of community-dwelling participants reporting a medical visit during the previous 3 months varied markedly, from $32.2 \%$ in Japan to $86.6 \%$ in France (table 1). The use of nursing care at home was uneven across countries too, ranging from $17.6 \%$ in Japan to $57.1 \%$ in Sweden. Home-delivered meals were reported rarely in Japan $(4.2 \%)$ but frequently in Denmark $(62.4 \%)$.

In contrast to variations in outpatient medical and nursing care, experiences of acute care hospital stays overnight were more homogenous and were similar among community-dwelling and institutionalised participants. The proportions reporting at least one stay in the previous year were lowest in France $(13.2 \%$ in the community, $12.9 \%$ in institutions), and highest in Sweden (28.3\% in the community) and Denmark (20.9\% in institutions).

Recent assessments of dental and oral health were mentioned by $31.3 \%$ of community-dwelling respondents in Japan-below the $56.0 \%$ observed in Sweden. The gap was wider among institutionalised centenarians (25.8\% in France to $65.9 \%$ in Sweden). Evaluations of vision and hearing showed more constant patterns: Japan exhibited the lowest values among communitydwellers (29.1\% and $12.2 \%$, respectively) and Switzerland the highest $(59.5 \%$ and $50.0 \%)$. Sensory testing was less frequent among institutionalised than among community-dwelling centenarians in all countries. In contrast, blood pressure measurement and weighing during the previous year were widespread in both community-dwelling and institutionalised participants, with the notable exception of Denmark where it was only reported by a minority. Finally, fewer than half the Japanese participants mentioned a recent evaluation of nursing care, home help and social care needs $(46.8 \%$ of community-dwellers and $44.9 \%$ of those institutionalised), whereas it was almost universal in Switzerland ( $83.7 \%$ and $100 \%$, respectively).

\section{Use of assistive devices}

Mobility habits differed across countries: the proportion of community-dwellers using no device or only a walking aid indoors ranged from $72.4 \%$ in Japan $(16.1 \%$ in institutions) to $97.0 \%$ in Denmark $(71.7 \%$ in institutions; table 2). Similarly, the share of community-dwelling respondents using no device or only a walking aid outdoors varied between $47.4 \%$ in Japan (9.8\% in institutions) and $96.6 \%$ in Denmark (60.8\% in institutions). More participants never went outdoors in Japan and Sweden $(6.9 \%$ and $10.7 \%$ in the community, respectively, $25.0 \%$ and $19.5 \%$ in institutions), with $14.3 \%$ of institutionalised Japanese respondents even being confined to bed. The proportion of community-dwellers using a mobility device to get around indoors (either a walking aid or a wheelchair) was uneven among countries too, ranging between $48.3 \%$ in Japan and $79.2 \%$ in Sweden. Participants living in institutions and reporting the use of a mobility device ranged from $78.6 \%$ in Japan to $98.2 \%$ in Denmark. Outdoors, the share of centenarians using mobility devices was usually over $75 \%$. There was a predominance of wheelchairs over walking aids in Japan, whereas the opposite was observed in Denmark.

Regarding other assistive devices, glasses $(77.3 \%$ of all participants) and dentures $(76.0 \%)$ were mentioned most frequently. Fewer than half respondents $(47.0 \%)$ had hearing aids. Overall, assistive devices were more commonly used in institutions than in the community, except for glasses and hearing aids. Percentages tended to be above average in Sweden and Denmark, irrespective of place of living, and below average in Japan. 


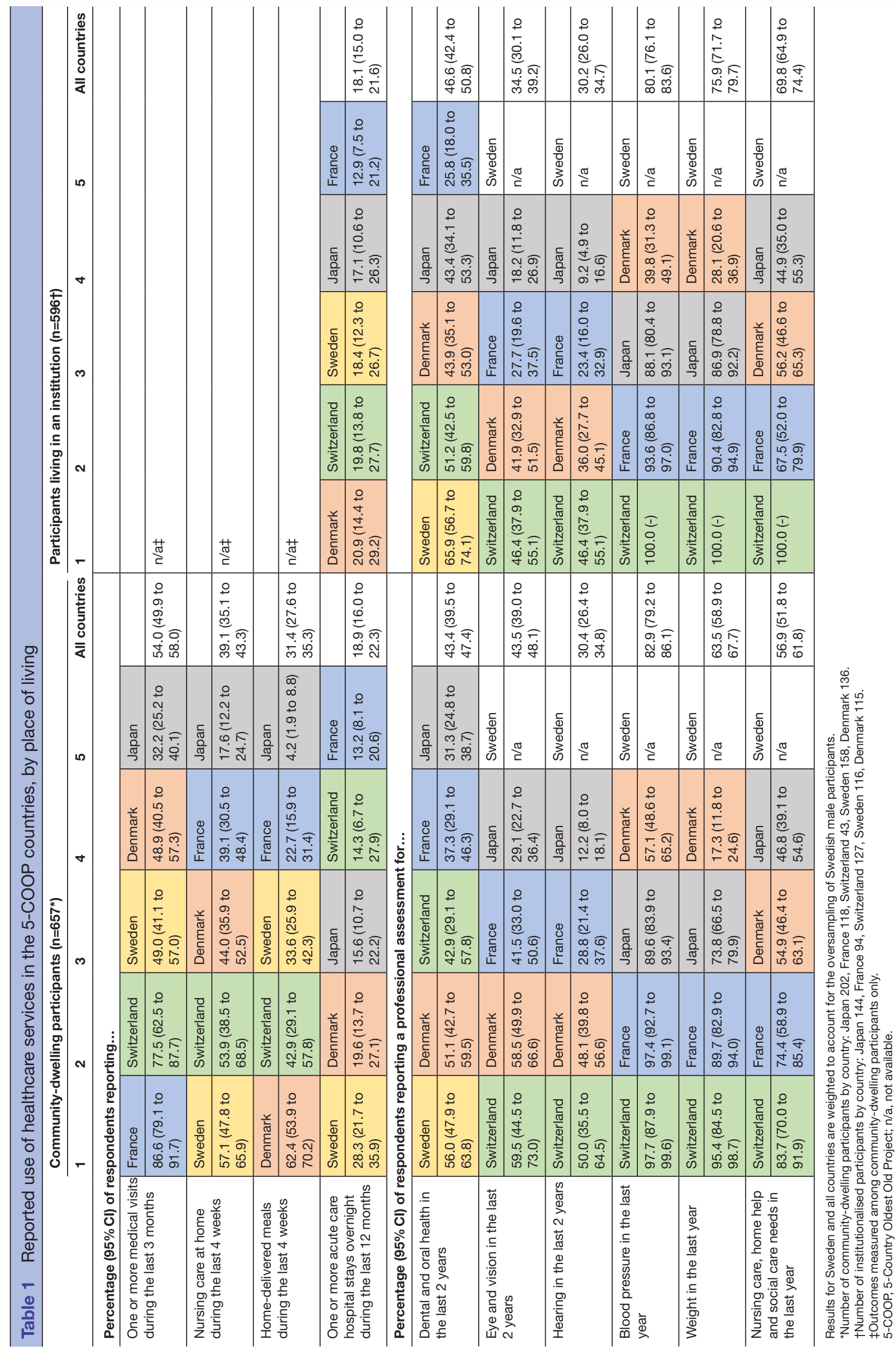




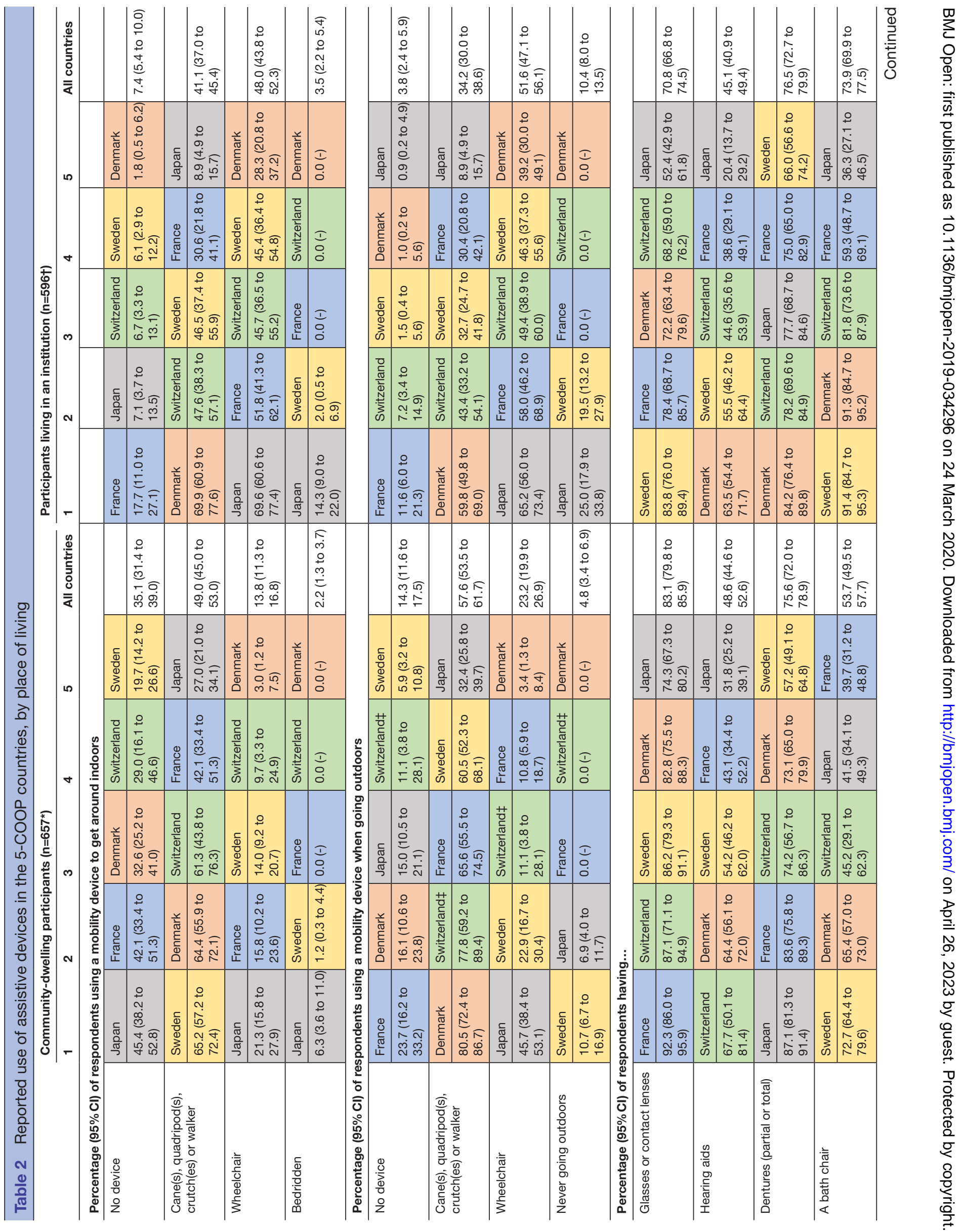




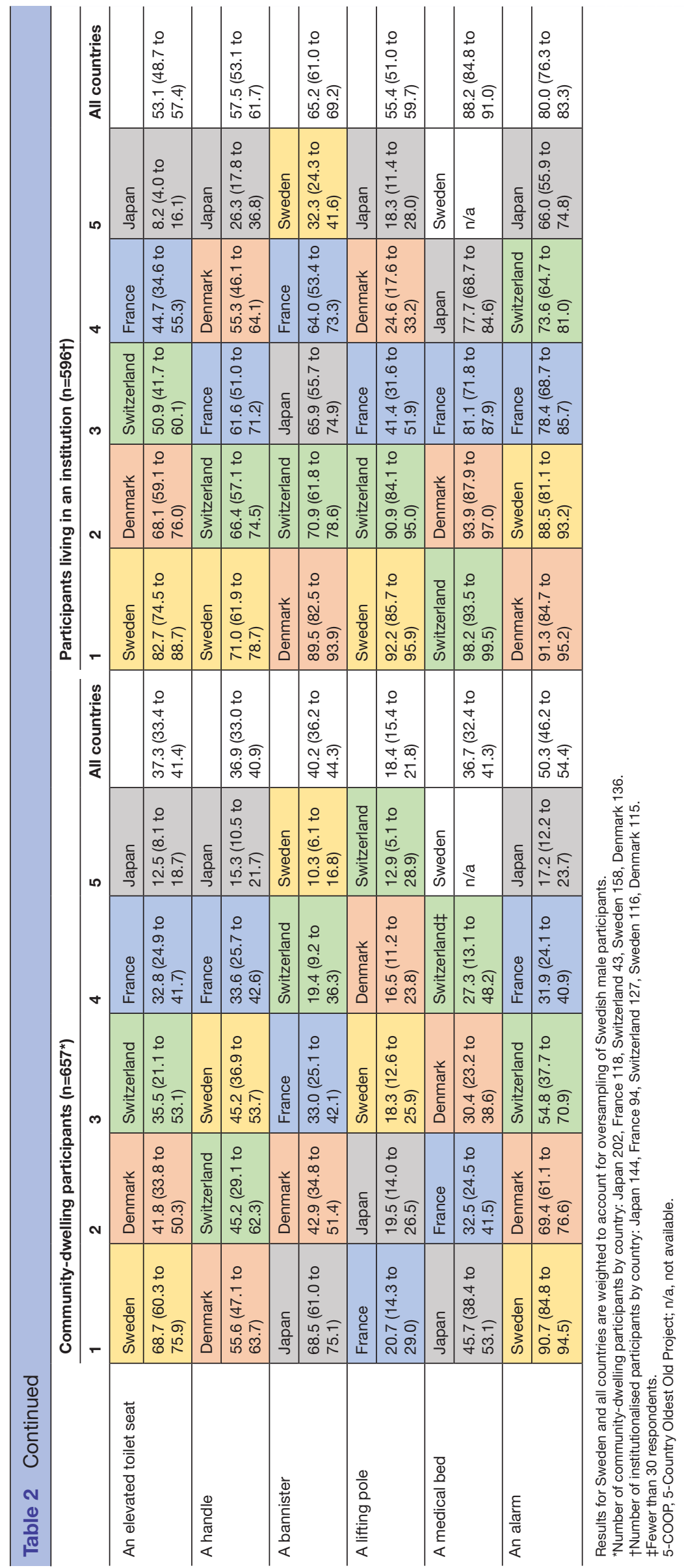




\section{Specific characteristics of national healthcare systems}

In 2015, centenarians were more than twice as common in Japan (45.5 per 100000 population) than in Switzerland, Sweden or Denmark (19.6, 19.1 and 20.0 per 100 000, respectively), with France lying in between (31.0 per 100000 ; table 3). Variation in the share of gross domestic product (GDP) devoted to healthcare was limited (from $10.3 \%$ in Denmark to $11.9 \%$ in Switzerland), but the proportion of total healthcare expenditure dedicated to long-term care differed from $14.9 \%$ in France to $26.2 \%$ in Sweden.

In terms of healthcare resources, the number of practising physicians ranged from 2.4 per 1000 population in Japan to 4.2 per 1000 in Sweden. Acute care hospital capacity showed the opposite trend with 2.3 beds per 1000 population in Sweden and 7.9 per 1000 in Japan, with the latter exhibiting an average length of stay almost three times that of other countries (16.5 vs 5.7 days). Regarding hospital use, Japan had the lowest rate of inpatient care discharges for the overall population (124.1 per 1000), and France had the highest (181.9 per 1000). This was in contradiction to the discharge rate observed for people aged $\geq 80$ years, which was lowest in France (419.0 per $1000)$ and highest in Sweden (638.5 per 1000).

According to national statistics, elders were more likely to receive long-term care at home in Switzerland (15.7\% of people aged $\geq 65$ years), even though more staff were devoted to home care in Japan (4.5 workers per 100 population aged $\geq 65$ years vs 2.8 per 100 in Switzerland). In institutions, the opposite was true, with greater human resources in Switzerland (5.3 workers per 100 population aged $\geq 65$ years) than in Japan (1.4 per 100). The number of beds in residential long-term care facilities was also highest in Switzerland (65.9 per 1000 population aged $\geq 65$ years) and lowest in Japan (24.3 per 1000), as was the proportion of the elder population living in institutions (5.9\% in Switzerland and $2.7 \%$ in Japan).

\section{DISCUSSION}

\section{Principal findings}

There was significant geographical variability with regard to the use of healthcare services and assistive devices by centenarians, and this also differed according to their place of living (in an institution or in the community). Even though all five countries dedicated comparable shares of GDP to healthcare, their healthcare resources and use varied, as recently highlighted in a study comparing the healthcare systems of high-income countries, including those involved in 5-COOP. ${ }^{12}$

\section{Analysis and comparison with other studies}

Apart from in Switzerland, the share of institutionalised centenarians in our sample agreed with the findings of several studies (from $42.1 \%$ in Portugal to $53.7 \%$ in Canada). ${ }^{9} 101314$ However, other works disclosed proportions above $60 \%$, and a study based on European census data revealed considerable geographical variations, with percentages ranging from $10 \%$ to $90 \% .^{61516}$ The Swiss exception observed in 5-COOP is consistent with its more substantial proportion of elders living in institutions, as reported in OECD statistics, and matches the greater numbers of staff and long-term care beds found there. Moreover, this is supported by the findings of recent research disclosing a high prevalence $(27.9 \%)$ of nursing home admissions in elders' last years of life in Switzerland. ${ }^{17}$ Institutionalisation did not seem to act as a substitute for home care, whose use was also more frequent in Switzerland, nor was it related to the proportion of total healthcare expenditure dedicated to long-term care.

The number of medical visits seemed to match differences in the number of general practitioners in each country, but the relationship was less noticeable when taking all practising physicians together. In other studies addressing the topic, $>95 \%$ of centenarians had seen a doctor during the previous 12 months. ${ }^{9} 1015$ Regarding nursing care at home, its more frequent use in Switzerland and Sweden was comparable to that reported in Germany $(52.8 \%)$ and Canada $(55.9 \%)$, whereas its use in France and Denmark was closer to that recently found in Portugal (44.4\%). ${ }^{9} 1013$ Japan distinguished itself by more limited use of nursing care at home. Our observations agreed with the higher shares of elderly populations who received home care in Switzerland and Sweden, but they appear paradoxical in light of greater staff resources devoted to home care in Japan. However, this could be skewed by the double-counting of Japanese workers who have two or more employers in the OECD statistics. ${ }^{18}$ The characteristics of healthcare systems do not explain divergent national practices regarding home-delivered meals, perhaps because this service is often provided by welfare services instead, as in Japan. ${ }^{19}$ Moreover, in Japan, meal preparation can also be part of the duties of paid home helpers. Disparities exist within countries too, as in Sweden, where municipalities are responsible for organising meals-on-wheels and pursue varying policies. ${ }^{20}$ In contrast, centenarians' experiences of acute care hospital stays overnight differed little between countries and were similar to other observations made in Europe, Canada and Australia (ranging from $10.9 \%$ to $22.1 \%$ ). ${ }^{6} 101415$ Studies conducted in England and the USA have revealed annual hospital admission rates for centenarians superior to $50 \%$, suggesting that all the 5-COOP countries had low hospitalisation rates by international standards. ${ }^{2122}$ It should be noted, however, that hospitalised centenarians were excluded by the 5-COOP investigators, which could have led to an underestimation of hospitalisation rates. Our results did not match differences in the number of hospital beds available in the participating countries. Interestingly though, our observations seem to evolve in parallel with the discharge rates for the population aged $\geq 80$ years but not with that of the general population, possibly revealing differing practices concerning the hospitalisation of the oldest-old.

Our results confirmed the limited access elders have to dental care and its variability from one country to 


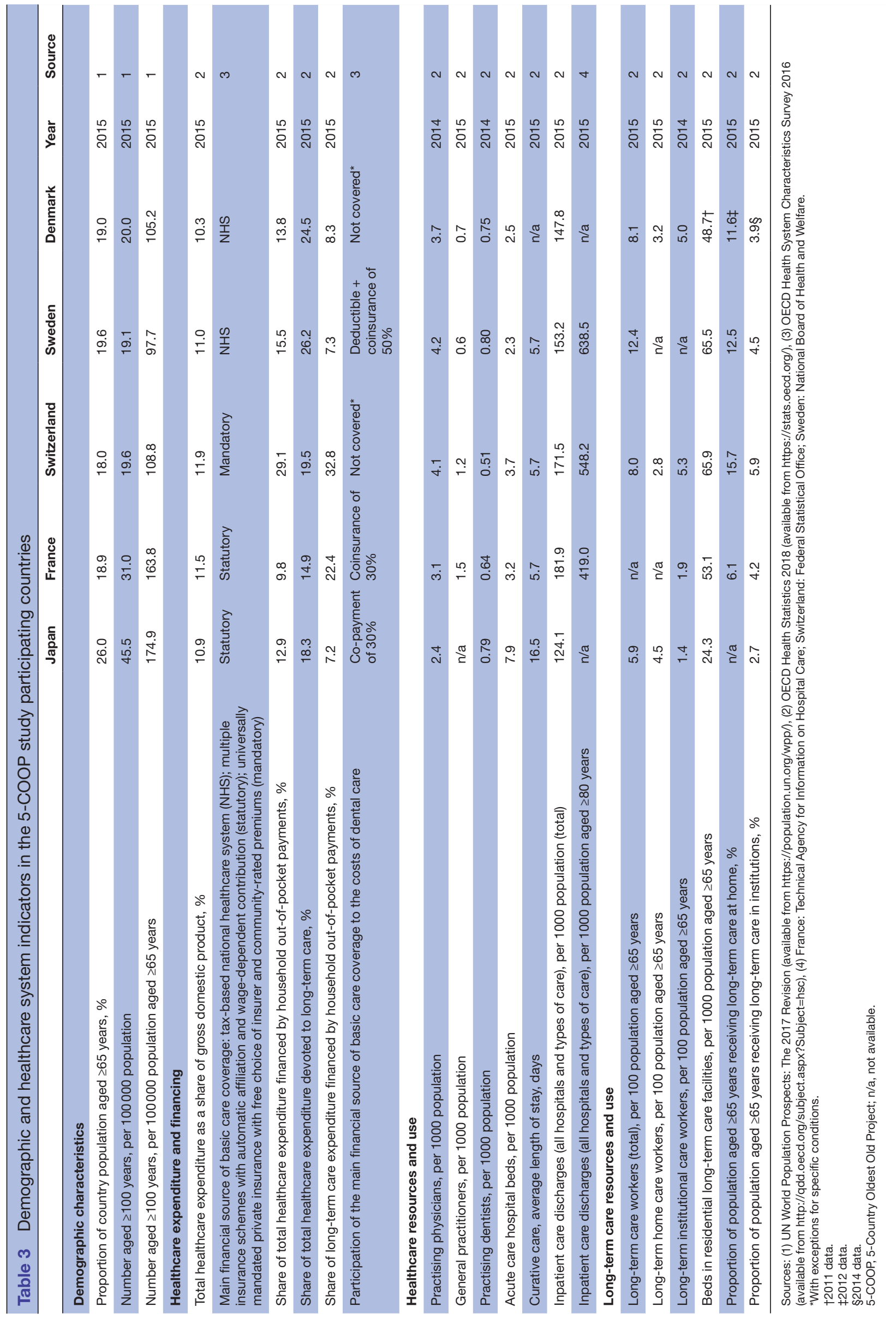


another (previously underscored by WHO) despite the well-recognised importance of oral health for quality of life and health status. ${ }^{23}$ Interestingly, insurance coverage for dental costs did not seem to have an influence, nor did the density of dentists. The share of participants reporting a sensory assessment was even lower than that observed for oral health, despite the widespread prevalence of vision and hearing deficiencies among centenarians and the negative impact they have on wellbeing. ${ }^{25-27}$ The contrast was striking in comparison to blood pressure and weight monitoring, which was almost generalised, even though its positive impact on quality of life is less obvious. Regarding assessments of nursing care, home help and social care needs, the higher numbers observed among community-dwellers in France and Switzerland could be related to those countries' wider use of medical visits and, in the case of Switzerland, of nursing care at home, both interventions being conducive to such assessments. However, given the evidence supporting the benefits of comprehensive assessments for elders, notably in terms of daily functioning, the variety of practices is surprising. ${ }^{28}$ In Swiss nursing homes, these evaluations fall under the healthcare reimbursement system, which explains their systematic use.

Few studies have addressed centenarians' use of assistive devices, and their results showed various tendencies, whether with mobility aids or other devices. ${ }^{13} 142930$ Although we were unable to draw any conclusions from the present study's settings, differences in the use of mobility aids could be related to differences in centenarians' functional limitations and influenced by cultural contexts too. Interestingly, in recent research based on the same study population, the prevalence of falls in Japan, which had the highest proportion of bedridden or wheelchair-using centenarians, was about half that of the other countries. ${ }^{31}$ However, it is known that socioeconomic aspects play a significant part too, and the common use of mobility devices in Sweden and Denmark could be related to them being heavily subsidised by the tax-based national healthcare system; higher out-of-pocket expenses may limit their acquisition in other countries. ${ }^{32}{ }^{33}$ Having glasses did not match the frequency of the vision assessments discussed earlier. On the contrary, countries providing more hearing evaluations showed broader hearing aid use, indicating that the limited access to such devices could be partly related to underdiagnosis. Interestingly, there seemed to be an inverse relationship between the frequency of dental assessments and the possession of dentures. It is open to debate whether limited access to dental care results in poor dentition and the use of dentures, or whether centenarians who still have their natural teeth are more likely to visit an oral health professional. As with mobility aids, the high frequency of use of devices such as hearing aids in Sweden and Denmark could result partly from more generous insurance coverage by their national healthcare systems.

\section{Study strengths and weaknesses}

The large sample of centenarians surveyed was a strength of the 5-COOP study and it was the first international investigation of healthcare services used by centenarians using a common survey questionnaire, thus permitting direct comparisons between countries at similar levels of economic development. Moreover, we were able to explore new fields, such as the delivery of meals-onwheels and the frequency of dental and sensory assessments, as well as the use of assistive devices, never before considered.

However, several limitations need to be acknowledged too. First, the varied participation rates, sampling methods and interview modes used could have differentially affected the representativeness of the participants in each country, hence biassing comparisons. We also cannot exclude that healthier centenarians were more prone to participate in the study, resulting in biassed estimations. Participation by proxies should have limited this possibility. Unfortunately, the available data did not allow us to assess the reliability of proxy interviews. Second, the methodology used in collecting OECD statistics is also subject to local specificities, which could jeopardise the comparability of indicators across nations. Third, differences in the use of healthcare services between countries may be partly explained by variations in the involvement of informal caregivers, a dimension which our indicators were unable to capture. In the same vein, differences in centenarians' health status could have contributed to variable use patterns, as suggested by the uneven prevalence of the frailty phenotype recently shown in another 5-COOP paper. ${ }^{8}$ However, health status and care habits are sometimes difficult to disentangle, and differences in use did not show any systematic patterns or match the prevalence of frailty. This suggests that if such a difference in health status existed, it would not account for all of the heterogeneity observed in the use of healthcare services and assistive devices.

\section{CONCLUSIONS}

Despite living in countries with comparable economic conditions and healthcare spending, the centenarians participating in the 5-COOP study reported varying use of healthcare services and assistive devices. Although causality remains to be established, some of these differences could be related to the characteristics of national healthcare systems, especially the type of healthcare insurance coverage and the amount of specific resources allotted to healthcare. However, unexplored factors may also be at work. Cultural differences, as in the role of informal caregivers, for example, could be significant. Our results revealed differing models of care, but whether one of those models is better suited to improving centenarians' health outcomes and quality of life remains an open question.

Author affiliations

${ }^{1}$ Center for Primary Care and Public Health (Unisanté), University of Lausanne, Lausanne, Switzerland 
${ }^{2}$ Epidemiology and Biostatistics, Institute of Public Health, and Danish Aging Research Centre, University of Southern Denmark, Odense, Denmark ${ }^{3}$ Department of Geriatrics, Odense University Hospital, Odense, Denmark ${ }^{4}$ Aging Research Center, Karolinska Institutet and Stockholm University, Stockholm, Sweden

${ }^{5}$ U1168, VIMA: Aging and Chronic Diseases, Epidemiological and Public Health Approaches, INSERM, Villejuif, France

${ }^{6}$ UMR-S 1168, Université de Versailles St-Quentin-en-Yvelines, Montigny le Bretonneux, France

${ }^{7}$ Département Hospitalier d'Epidémiologie et Santé Publique, Hôpitaux Universitaires Paris Ile-de-France Ouest, Assistance Publique-Hôpitaux de Paris, Paris, France ${ }^{8}$ Division of Geriatrics, Department of Rehabilitation and Geriatrics, Geneva University Hospitals and University of Geneva, Geneva, Switzerland

${ }^{9}$ Tokyo Metropolitan Institute of Gerontology, Tokyo, Japan

${ }^{10}$ MMDN, Université de Montpellier, EPHE, INSERM, U1198, PSL Research University, Montpellier, France

${ }^{11}$ College of Economics and Population Research Institute, Nihon University, Tokyo, Japan

Acknowledgements The authors would like to thank all study participants and their close relatives for their involvement.

Collaborators The 5-COOP group includes Kaare Christensen and Anna Oksuzyan (University of Southern Denmark), Frederic Balard (INSERM), Yasumichi Arai and Nobuyoshi Hirose (Keio University), Yukie Masui and Hiroki Inagaki (Tokyo Metropolitan Institute of Gerontology), Yasuyuki Gondo and Kei Kamide (Osaka University), Craig Willcox, Ikuya Ashitomi and Shotoku Yasura (Okinawa International University), Makoto Suzuki (University of the Ryukyus), Marti Parker (deceased), Mats Thorslund (retired), Bettina Meinow (Karolinska Institutet \& Stockholm University), Fred Paccaud (retired), and Dina Zekri (Hôpitaux Universitaires de Genève).

Contributors JD did the statistical analyses and drafted the manuscript. JD and $B S-E$ designed the article and contributed to the interpretation of data. JD, KA-R, $\mathrm{SF}, \mathrm{MH}$ and TW collected national healthcare system indicators. FRH gathered and prepared 5-COOP data for analysis. KA-R, SF, MH, FRH, BJ, J-MR, YS, BS-E and the other members of the 5-COOP group contributed to the conception of the survey questionnaire. KA-R, SF, MH, FRH, BJ, J-MR, YS and the other members of the 5-COOP group participated in planning of the 5-COOP study and collection of data. All authors commented on drafts, read and approved the final manuscript. The corresponding author attests that all listed authors meet authorship criteria and that no others meeting the criteria have been omitted.

Funding Funding of the present research was provided by the operating budget of the Center for Primary Care and Public Health (Unisanté), University of Lausanne, Switzerland. Three preparatory international seminars of the 5-COOP study were supported by the CERA (Caisse d'Epargne Rhône-Alpes) Foundation. Data collection was supported by the AXA Research Fund. The conception of the 5-COOP study was independent from the sponsors.

Competing interests None declared.

Patient and public involvement Patients and/or the public were not involved in the design, conduct, reporting or dissemination plans of this research.

\section{Patient consent for publication Not required.}

Ethics approval 5-COOP study was approved by the following ethics committees: Japan: Nihon University (ID 23-9), Okinawa International University (ID 申-10), Keio University (ID 2011-155), Tokyo Metropolitan Institute of Gerontology (ID 58), Osaka University (ID 23022). France: Comité de Protection des Personnes SudMéditerranée IV (ID 2010-A01510-39), Agence française de sécurité sanitaire des produits de santé (ID B110089-20), Commission Nationale de l'Informatique et des Libertés (ID 1493186 v 0) Switzerland: Geneva Canton (ID 11-037/Psy 11-004), Vaud Canton (ID 339/2013), Fribourg Canton (ID 339/2013 addendum), Jura Canton (ID 340/2013), Neuchâtel Canton (ID 341/2013), Valais Canton (ID 021/14). Sweden: Regional Ethical Review Board in Stockholm (ID 2011/306-31/3). Denmark: Committee on Health Research Ethics (ID S-20110011). Data were collected after obtaining the consent of the participants.

Provenance and peer review Not commissioned; externally peer reviewed.

Data availability statement The data supporting the findings of this study are available within the article and its supplementary material.

Open access This is an open access article distributed in accordance with the Creative Commons Attribution Non Commercial (CC BY-NC 4.0) license, which permits others to distribute, remix, adapt, build upon this work non-commercially, and license their derivative works on different terms, provided the original work is properly cited, appropriate credit is given, any changes made indicated, and the use is non-commercial. See: http://creativecommons.org/licenses/by-nc/4.0/.

ORCID iD

Julien Dupraz http://orcid.org/0000-0003-0082-8759

\section{REFERENCES}

1 United Nations,, Department of Economic and Social Affairs,, Population Division. World Population Prospects: The 2017 Revision, Key Findings and Advance Tables. Working Paper No. ESA/P/ WP/248. [Internet], 2017. Available: https://esa.un.org/unpd/wpp/ Publications/Files/WPP2017_KeyFindings.pdf

2 Christensen K, Doblhammer G, Rau R, et al. Ageing populations: the challenges ahead. Lancet 2009;374:1196-208.

3 Robine J-M, Cubaynes S. Worldwide demography of centenarians. Mech Ageing Dev 2017;165:59-67.

4 Willcox DC, Willcox BJ, Poon LW. Centenarian studies: important contributors to our understanding of the aging process and longevity. Curr Gerontol Geriatr Res 2010;2010:484529.

5 Jopp DS, Boerner K, Ribeiro O, et al. Life at age 100: an international research agenda for centenarian studies. J Aging Soc Policy 2016;28:133-47.

6 Tettamanti M, Marcon G. Cohort profile: 'Centenari a Trieste' (CaT), a study of the health status of centenarians in a small defined area of Italy. BMJ Open 2018;8:e019250.

7 Robine J-M, Cheung SLK, Saito Y, et al. Centenarians today: new insights on selection from the 5-COOP study. Curr Gerontol Geriatr Res 2010;2010:120354.

8 Herr M, Jeune B, Fors S, et al. Frailty and associated factors among centenarians in the 5-COOP countries. Gerontology 2018;64:521-31.

9 von Berenberg P, Dräger D, Zahn T, et al. Chronic conditions and use of health care service among German centenarians. Age Ageing 2017;46:939-45.

10 Rochon PA, Gruneir A, Wu W, et al. Demographic characteristics and healthcare use of centenarians: a population-based cohort study. $J$ Am Geriatr Soc 2014;62:86-93.

11 Brandão D, Ribeiro O, Freitas A, et al. Hospital admissions by the oldest old: past trends in one of the most ageing countries in the world. Geriatr Gerontol Int 2017;17:2255-65.

12 Papanicolas I, Woskie LR, Jha AK. Health care spending in the United States and other high-income countries. JAMA 2018;319:1024-39.

13 Ribeiro O, Araújo L, Teixeira L, et al. Health status, living arrangements, and service use at 100: findings from the Oporto centenarian study. J Aging Soc Policy 2016;28:148-64.

14 Afonso RM, Ribeiro O, Vaz Patto M, et al. Reaching 100 in the countryside: health profile and living circumstances of Portuguese centenarians from the Beira interior region. Curr Gerontol Geriatr Res 2018;2018:8450468.

15 Roughead EE, Kalisch LM, Ramsay EN, et al. Use of health services and medicines amongst Australian war veterans: a comparison of young elderly, near centenarians and centenarians. BMC Geriatr 2010;10:83.

16 Teixeira L, Araújo L, Jopp D, et al. Centenarians in Europe. Maturitas 2017;104:90-5.

17 Stolz E, Mayerl H, Rásky Éva, et al. Individual and country-level determinants of nursing home admission in the last year of life in Europe. PLoS One 2019;14:e0213787.

18 OECD. OECD Health Statistics 2018: Definitions, Sources and Methods [Internet], 2018. Available: http://www.oecd.org/health/ health-data.htm

19 Yanagisawa $\mathrm{H}$, Sakakibara $\mathrm{H}$. Factors affecting satisfaction levels of Japanese volunteers in meal delivery services for the elderly. Public Health Nurs 2008:25:471-9.

20 Skinnars Josefsson M, Nydahl M, Persson I, et al. Reforming foodservice in elderly care: national actions and local outcomes. Nutr Diet 2018;75:79-86.

21 Dotchin CL, Gray WK, Gaskin E, et al. Frequency, nature and outcomes of hospital admissions in centenarians in an area of NorthEast England. Geriatr Gerontol Int 2016;16:969-75.

22 Mandawat A, Mandawat A, Mandawat MK, et al. Hospitalization rates and in-hospital mortality among centenarians. Arch Intern Med 2012:172:1179-80.

23 Petersen PE, Kandelman D, Arpin S, et al. Global oral health of older people-call for public health action. Community Dent Health 2010;27:257-67. 
24 Müller F, Shimazaki Y, Kahabuka F, et al. Oral health for an ageing population: the importance of a natural dentition in older adults. Int Dent J 2017;67:7-13.

25 Crews JE, Campbell VA. Vision impairment and hearing loss among community-dwelling older Americans: implications for health and functioning. Am J Public Health 2004;94:823-9.

$26 \mathrm{Li} \mathrm{J}$, Zhao $\mathrm{H}$, Huang B, et al. A survey on hearing acuity of centenarians in Hainan Province. J Otol 2018;13:135-7.

27 Toyoshima A, Martin P, Sato S, et al. The relationship between vision impairment and well-being among centenarians: findings from the Georgia centenarian study. Int J Geriatr Psychiatry 2018;33:414-22.

28 Stuck AE, Siu AL, Wieland GD, et al. Comprehensive geriatric assessment: a meta-analysis of controlled trials. Lancet 1993;342:1032-6.

29 Rasmussen SH, Thinggaard M, Højgaard MB, et al. Improvement in Activities of Daily Living Among Danish Centenarians? - A
Comparative Study of Two Centenarian Cohorts Born 20 Years Apart. J Gerontol A Biol Sci Med Sci 2018;73:1125-31.

30 Engberg $\mathrm{H}$, Christensen K, Andersen-Ranberg K, et al. Improving Activities of Daily Living in Danish Centenarians--But Only in Women: A Comparative Study of Two Birth Cohorts Born in 1895 and 1905. J Gerontol Ser A 2008;63:1186-92.

31 Chen T-Y, Chan A, Andersen-Ranberg K, et al. Prevalence and correlates of falls among centenarians: results from the Five-Country oldest old project (5-COOP). J Gerontol A Biol Sci Med Sci 2019;360.

32 Clarke P, Chan P, Santaguida PL, et al. The use of mobility devices among institutionalized older adults. J Aging Health 2009;21:611-26.

33 Mathieson KM, Kronenfeld JJ, Keith VM. Maintaining functional independence in elderly adults: the roles of health status and financial resources in predicting home modifications and use of mobility equipment. The Gerontologist 2002;42:24-31. 